\title{
Context-Aware POI Sequence Recommendation with Attention-Based Neural Network
}

\author{
Jianfeng Huang ${ }^{\text {a }}$, Yuefeng Liu ${ }^{\mathrm{a}, *}$, Yue Chen ${ }^{\mathrm{a}}$, Chen Jia ${ }^{\mathrm{a}}$ \\ a School of Earth and Space Sciences, Peking University, P. R. China. Jianfeng Huang, jainfenghuang@pku.edu.cn, Yuefeng Liu, \\ yuefengliu@pku.edu.cn, Yue Chen, chenyue_sess@pku.edu.cn, Chen Jia, jiachen07@pku.edu.cn \\ * Corresponding author
}

Keywords: POI Sequence recommendation, Attention-Based Neural Network, Location-based social networks

\begin{abstract}
:
Location-based social networks (LBSNs) is playing an increasingly important role in our daily life, through which users can share their locations and location-related contents at any time. The Location information implicitly expresses user's behaviour preference. Therefore, LBSNs is being widely explored for Point-of-Interest (POI) recommendation in recent years. Most of existing POI recommenders only recommend a single POI, while sometimes successive POI sequence recommendation is more practical. For example, when we travel to a strange city, what we expect is not a single POI recommendation, but a POI sequence recommendation which contains a set of POIs and the order of visiting them. To solve this problem, this paper proposes a novel model called Context-Aware POI Sequence Recommendation (CPSR), which is developed based on an attention-based neural network. Neural network has made a great success in various of field because of its powerful learning ability. Recently, dozens of works has demonstrated that attention mechanism can make the neural network models more reasonable.
\end{abstract}

We define the POI sequence recommendation task as following: given a user's historical POI sequence $\left(h_{1}, h_{2}, h_{3}, \ldots, h_{n}\right)$, we need to recommend the next contextually coherent POI sequence $\left(p_{1}, p_{2}, p_{3}, \ldots, p_{k}\right)$ for the user. Actually, it is a sequence to sequence (seq2seq) learning task. User's preferences are implicit in the context of sequence. Enlightened by many neural sequence transduction models, we design the CPSR based on an encoder-decoder structure (Figure 1), which has strong power to capture the contextual information in sequence. The encoder will learn the contextual information implied in the input sequence $\left(h_{1}, h_{2}, h_{3}, \ldots, h_{n}\right)$, and the contextual information is represent as a continuous vector $\boldsymbol{C}=\left(c_{1}, c_{2}, c_{3}, \ldots, c_{m}\right)$. Given $\boldsymbol{C}$, the encoder then generates the recommended POI sequence one by one. When generating the next POI, decoder also takes the previously generated POIs as additional input.

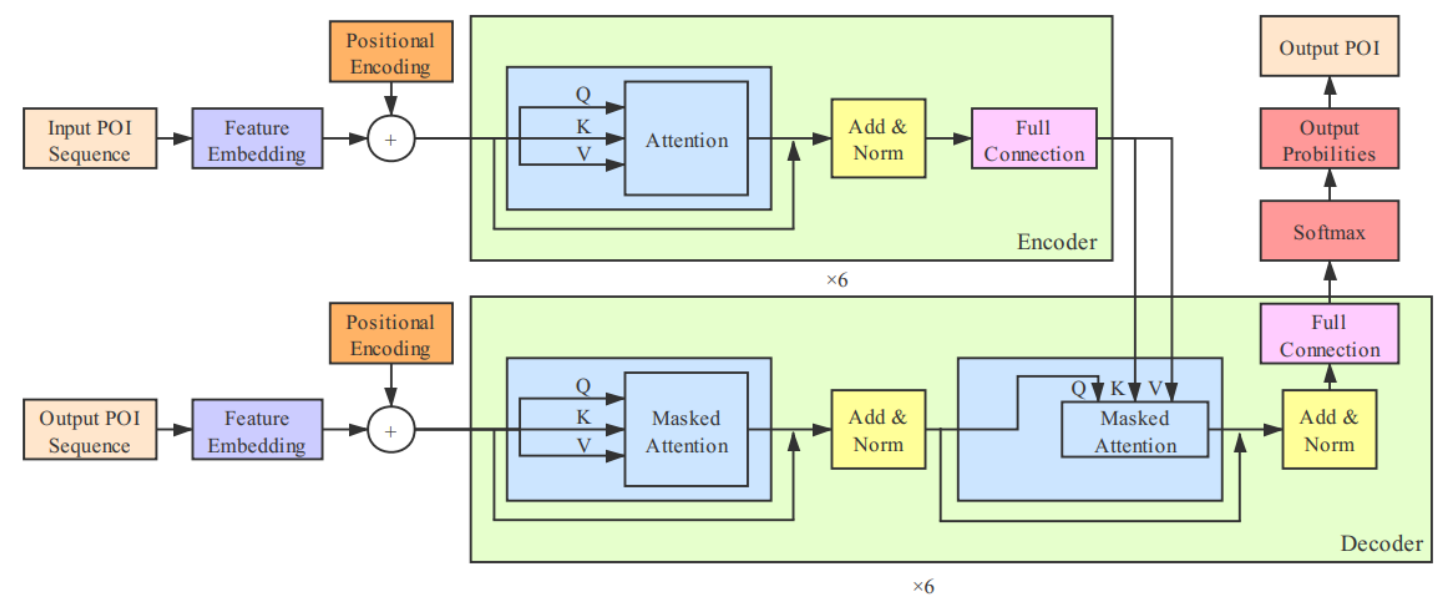

Figure 1. The architecture of CPSR.

More specifically, because each POI is originally represented as a text name, we first need to embedding each POI to a feature vector which should expresses many features of this POI such as geographical feature, category feature, and popularity feature. In this work, the feature embedding is carry out through a learnable way. In order to utilize the order information in the POI sequence, we add "positional encoding" into the CPSR. The "positional encoding" is calculated by formula (1):

$$
P E_{(p o s, 2 i)}=\sin \left(\frac{p o s}{1000^{\frac{2 i}{d}}}\right), \quad P E_{(p o s, 2 i+1)}=\cos \left(\frac{p o s}{1000^{\frac{2 i}{d}}}\right)
$$


where pos denotes the position of a POI, $i$ denotes the $i$-th dimension, and the $d$ is the dimension of the positional encodings. The encoder consists of a stack of 6 identical blocks, and each block has an attention layer and a fully connected layer. The attention layer is calculated by formula (2):

$$
\operatorname{Attention}(Q, K, V)=\operatorname{softmax}\left(\frac{Q K^{T}}{\sqrt{d_{k}}}\right) V,
$$

where $Q, K$, and $V$ are the embedded features, $d_{k}$ is the dimension of $K$. Similar to encoder, the decoder consists of a stack of 6 identical blocks. Besides the two sub-layer in each encoder block, decoder insert a third sub-layer, which takes the output of encoder as input. Finally, the output of decoder is transformed to a probability distribution through a softmax layer, then the POI with the maximum probability will be recommended in current step. Note that the previously generated POIs will be taken as input (i.e. the "output POI sequence" shown in Figure 1) when generating next POI.

In the experiments, we validate the CPSR on the Weeplaces dataset. This dataset is collected from Weeplaces, a website that aims to visualize users' check-in activities in LBSNs. We extract all the data located in New York for experiments, which contains 639,931 check-ins generated by 2,161 users over 8,958 locations. Based on the dataset, each user's historical POI sequence is divided into a series of samples like $\left(\left[h_{1}, h_{2}, h_{3}, \ldots, h_{n}\right],\left[g_{1}, g_{2}, g_{3}, \ldots, g_{k}\right]\right)$. Here we set the length of input POI sequence $n$ as 30, and set the length of recommended POI sequence $k$ as 5 . We respectively select $50 \%$ and $80 \%$ of samples for training, correspondingly, $50 \%$ and $20 \%$ for testing.

To evaluate the accuracy of the recommended POI sequence, we define the metrics Align-Precision (AP) as:

$$
A P(\boldsymbol{G}, \boldsymbol{P})=\frac{\sum_{i=1}^{k} I\left(\boldsymbol{G}_{i}, \boldsymbol{P}_{i}\right)}{k}
$$

where $k$ is the length of the recommended POI sequence, and if $\boldsymbol{G}_{i}=\boldsymbol{P}_{i}, I\left(\boldsymbol{G}_{i}, \boldsymbol{P}_{i}\right)=1$, otherwise $I\left(\boldsymbol{G}_{i}, \boldsymbol{P}_{i}\right)=0$. For example, $A P([1,2,3,4,5],[1,6,3,8,5])=0.6$. The experimental results have been presented in Table 1 . The results show that CPSR has great potential in POI sequence recommendation task.

\begin{tabular}{ccc}
\hline Training set:Testing set & $50 \%: 50 \%$ & $80 \%: 20 \%$ \\
\hline AP & 0.3124 & 0.4342 \\
\hline
\end{tabular}

Table 1. The AP on testing set under different setting.

Figure 2 shows an example of POI sequence recommendation, where the AP is 0.8 .

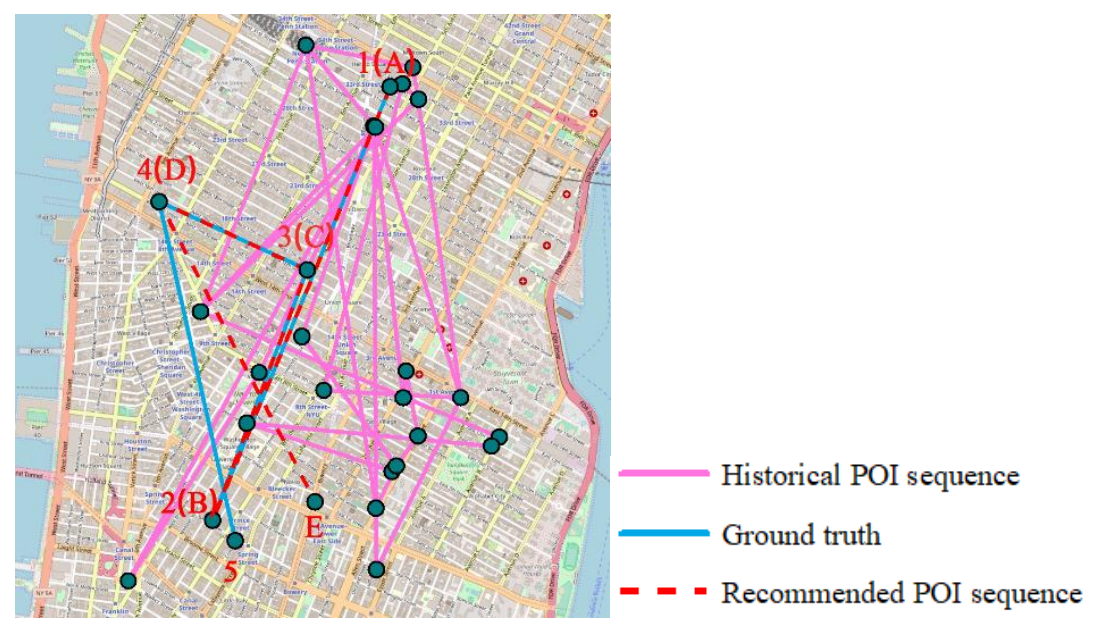

Figure 2. An example of POI sequence recommendation. The number 1-5 denote the ground truth, and the letter A-E denote the recommended POI sequence.

Itinerary planning is a time-consuming process, because we always need to consider so many constraints such as time, distance, preference, etc. Therefore, a POI sequence recommender is particularly necessary. Based on the attentionbased neural network, a novel framework called CPSR is proposed for the POI sequence recommendation task in this paper. CPSR first learns the contextual information of sequence through encoder, then generates recommended POI sequence through decoder. And the experiments demonstrate the effectiveness of CPSR. Moreover, other information implicit in LBSNs such as social relations may be helpful for POI sequence recommendation, which is what we will explore in the future works. 